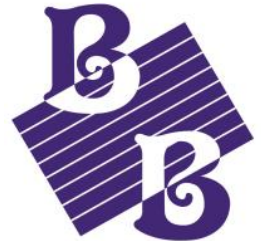

BioBacta
Journal of Bioscience and Applied Research

www.jbaar.org

\title{
Neutrophil gelatinase-associated lipocalin and oxidative stress markers based -scores to improve the diagnostic accuracy of chronic kidney diseases
}

\author{
Toson el-SA ${ }^{1}$, Waly $\mathbf{S}^{1}$, Omran $\mathbf{M M}^{2, *}$ \\ ${ }^{1}$ Chemistry Department, Faculty of Science, Damietta University, Damietta, Egypt \\ ${ }^{2}$ Chemistry Department, Faculty of Science, Helwan University, Cairo, Egypt \\ *Correspondence: Mohamed Mostafa Omran, PhD Chemistry Department, Faculty of Science, \\ Helwan University, Ain Helwan, 11795 Cairo, Egypt, E-mails: drmmomran@yahoo.com
}

Brief tittle: Novel scores for improving the diagnosis of chronic kidney diseases Received date: December 20, 2018. Accepted: January 21, 2019

\begin{abstract}
Background: Biomarkers can detect chronic kidney disease (CKD) in an early stage. Patients and methods: 75 individuals suffering from chronic kidney disorders were included in this study. Serum Neutrophil gelatinaseassociated lipocalin (NGAL) was assayed using ELISA. Nitric oxide (NO) in addition to total antioxidant capacity (TAC) was colorimetrically measured. Results: NGAL was the most efficient marker among others. Its area under the receiver operating characteristic (ROC) were 0.91 and 0.80 for differentiating patients with CKD from control individuals and discriminating patients in late stages from those in early stages, respectively. The stepwise multivariate discriminant analysis (MDA) selected two novel indexes. The first is to differentiate patients in late stages from those in early stages of CKD; namely NGAL- Total antioxidant capacity (NT) score. The second is to differentiate all of these patients from those of the control subjects; namely NGAL- nitric oxide (NN) score. The AUCs of NT and NN scores were 0. 84 and 0.93; respectively. Conclusion: NT and NN scores may add more in the evaluation and prediction of the progression of CKD.
\end{abstract}

Keywords: Kidney diseases, diagnosis, accuracy, neutrophil gelatinase-associated lipocalin, nitric oxide, and total antioxidant capacity. 


\section{Introduction}

Chronic kidney disease has recently presumed epidemic proportions, becoming a disturbing emerging cause of morbidity, especially if it proceeds to renal failure (Bolignano et al., 2009). Clinically; NGAL is a promising kidney injury biomarker (Bolignano et al., 2008). It is a $25-\mathrm{kD}$ a protein covalently tied to neutrophil gelatinase (Kjeldsen et al., 1993), belongs to the "lipocalins" superfamily, and is widely released in blood and urine from injured tubular cells after potential harmfulness to the kidney in experimental and human clinical examples. No less important, after damage NGAL releases from renal tubule, as an early predictor, before the serum creatinine rises and thus allowing starting the treatment as early as one can (Mori and Nakao, 2007). The source of nitric oxide (NO) production is by NO synthase (NOS) originated from endothelium with L-arginine-based mechanism (Wever et al., 1999). Therefore, L-arginine deficiency, decreased enzyme activity, and reduction in the important cofactor's availability can repress NO productivity. Changes in the expression of endothelial NOS (eNOS) and inducible NOS (iNOS) in the kidney diseases yields sustained afferent arteriolar vasoconstriction and tissue hypoxia (Thambyrajah et al., 2000). It was detected that NO production is decreased in CKD (Baylis, 2008). Also, the production of reactive oxygen species (ROS) as well as apoptosis mediates the progression of the disease to its late form. Oxidative stress and antioxidant depletion; including total antioxidant capacity (TAC), as a consequence of impaired mitochondrial respiratory system was noted in CKD patients. The product of excessive oxygen metabolism can not only disturb the nature of the lipid content of the cellular membrane, cell proteins, and nucleic acids but also, the cell function as well. They also mediate enzymatic inhibition of the cellular respiratory reaction (Modaresi et al., 2015). In the current study, we estimate and compare the diagnostic accuracy of the three biochemical markers; namely, NGAL, TAC, and NO to assess and predict the progression of CKD.

\section{Materials and methods}

Patients with different degrees $(n=75)$ of renal impairment referred to the CKD from the outpatient clinic of the Department of Internal Medicine of Alazhar University Hospital (March to June 2016) were examined. Regarding the distribution of CKD stages (Levey et al., 2009) the CKD patients were divided into stage1 with normal or high glomerular filtration rate (GFR) (GFR $>90 \mathrm{ml} / \mathrm{min}, \mathrm{S} 1$ and $\mathrm{n}=10)$, stage 2 mild CKD (GFR $=60-89 \mathrm{ml} / \mathrm{min}, \quad \mathrm{S} 2$ and $\mathrm{n}=14)$, stage 3 moderate CKD (GFR $=30-59 \mathrm{ml} / \mathrm{min}$, S3 and $n=26)$, stage4 Severe CKD (GFR $=15-29$ $\mathrm{ml} / \mathrm{min}$, S4 and $\mathrm{n}=13$ ) and stage 5 end Stage CKD (GFR < $15 \mathrm{ml} / \mathrm{min}$, S5 and $\mathrm{n}=12$ ) ( 8 patients under dialysis and 4 patients without dialysis). The first 3 stages (S1-S2-S3) were named as the early stages $(n=51)$ and the stages (S4-S5) were named as the late stages $(n=24)$. Further, healthy participants with normal kidney functions were used as a control group ( $n=25)$. Patients' history was carefully recorded by checking patients' records, drug prescriptions; clinical examination, blood pressure, diabetes, and $\mathrm{HCV}$ infection were performed. The study was approved by the local ethical committee. All participants were informed of the nature of the protocol and gave consent.

\section{Biochemical measurements}

Fasting venous blood samples were drawn, samples were centrifuged within 20 minutes, serum was separated and either freshly used or stored at $-70{ }^{\circ} \mathrm{C}$ for subsequent analysis. NGAL was measured in the blood using a commercially available ELISA kit (Cusabio, China), according to the manufacturer's prescript. Serum nitric oxide and total antioxidant capacity were measured using a Bio-diagnostic kit (Egypt), according to the manufacturer's prescript. Serum creatinine, urea, and albumin were measured using standard colorimetric techniques (Diamond diagnostics (Egypt), according to their enclosed pamphlets). Uric acid was measured using (a Spinreact kit, according to its enclosed pamphlets).

\section{Statistical analysis}


All results in this study were expressed as Mean \pm SE. ANOVA test was used to analyze the data by (SPSS) program, version 15. To compare continuous variables, ANOVA test, t-test or the non-parametric Mann-Whitney U test was used. The chi-square test or Fisher's exact test were used for categorical variables. The associations between the two markers were evaluated by Pearson's correlation coefficient. The multivariate discriminant analysis was stepped wisely done by using the minimum Wilk's lambda. A single model with the fewest variables and the greatest area under the receiver operating characteristic (ROC) was chosen. The accuracy as well as sensitivity (Sn), specificity (Sp), the predictive value of positive (PVP), and predictive value of negative (PVN) were evaluated.

\section{Results}

\section{Levels of lab parameters and candidate markers in patients with chronic kidney disease.}

The level of NGAL was significantly increased with increased severity of chronic kidney disease. NGAL level was about threefold higher in patients' group than that in the control group ( $\mathrm{p}<0.0001)$. NGAL concentration was 1.5 fold higher in late stages than that in the early stages $(\mathrm{p}<0.0001)$. NGAL concentrations were 4 and 3.5 -fold higher in stages 5 and 4 respectively, than that of the control group ( $\mathrm{p}$ $<0.0001)$. On the other hand, the levels of both TAC and $\mathrm{NO}$ were indirectly proportional to the progression of CKD stages. NO concentration was significantly decreased by $87 \%$ in stage 4 than that in the control group ( $\mathrm{P}<0.0001)$ but it was re-increased in stage 5. A decrease in TAC concentration was noted in stage 5 by $19 \%$ and in stage 4 by $12 \%$ than that in the control group ( $\mathrm{p}=0.006-0.01)$; respectively. TAC level was diminished by $16 \%$ in late stages compared to early stages $(p<0.005)$, whereas NO concentration was diminished around $62 \%$ in late stages compared to early stages $(p<0.0001)$. Further, a decrease in the GFR level was noted in the late stages by $74 \%$ compared to the early stages $(\mathrm{p}<0.0001)$. Albumin concentration was also lower in the late stages, which decreased by $10 \%$ compared to early stages $(\mathrm{p}=0.1)$. Levels of creatinine, uric acid, and blood urea nitrogen (BUN) were significantly increased in patients with CKD compared to the control group $(\mathrm{p}=0.002-\mathrm{p}<$ 0.0001); respectively. Patients, the group had Creatinine 4.4-fold higher than that in the control group ( $\mathrm{p}=0.002$ ) while BUN and uric acid had 2.3 and 1.5-fold higher in patients, group than that in the control group ( $p<0.0001)$; respectively (Table1). On the other hand, the levels of GFR ( $p<0.0001)$, Alb $(\mathrm{p}<0.0001)$, TAC $(\mathrm{p}=0.1)$ and NO $(\mathrm{p}<0.0001)$ were decreased by $63 \%, 16 \%, 8 \%$ and $61 \%$ in patients' group than those in the control group; respectively. It was noted that NGAL, NO, and TAC were independent variables for the prediction of chronic kidney disease (CKD) (Table 2). 
Table 1. Comparison among healthy control and chronic kidney disease patients in studied groups.

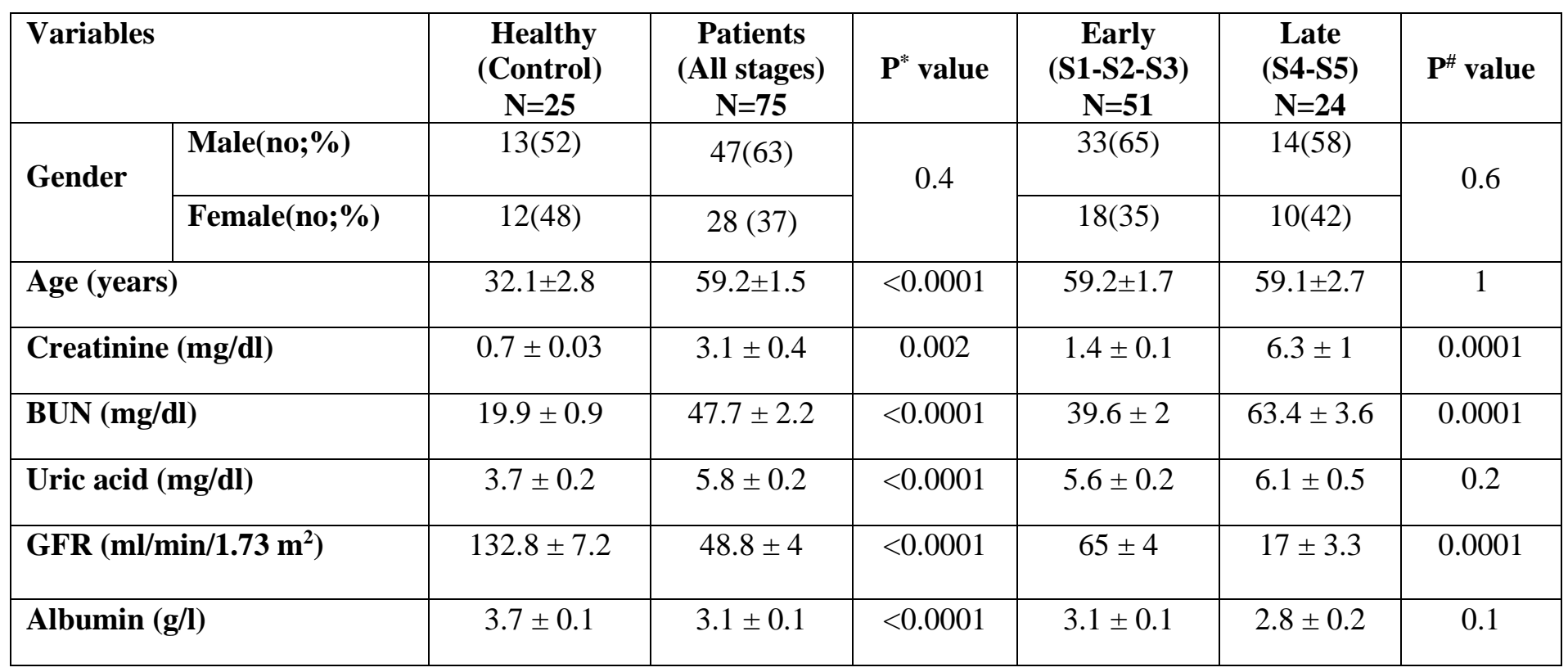

Values were expressed as mean \pm SE. BUN: Blood Urea Nitrogen. GFR: glomerular filtration rate. P value: $\mathrm{P}>0.05$ non-significant, $\mathrm{P}<0.05$ : Significant, $\mathrm{P}<0.001$ : very significant and $\mathrm{P}<0.0001$ : Extremely significant. $\mathrm{p}^{*}$ : $\mathrm{p}$ value of comparison between control versus patients' group. $\mathrm{P}^{\#}$ : $\mathrm{p}$ value of comparison between early stages versus late stages. 
Table2. The levels of Neutrophil gelatinase-associated lipocalin (NGAL), nitric oxide (NO) and total anti- oxidant capacity (TAC) in studied groups.

\begin{tabular}{|c|c|c|c|}
\hline Group & NGAL & NO & TAC \\
& $(\mathrm{ng} / \mathrm{ml})$ & $(\mu \mathrm{mol} / \mathrm{l})$ & $(\mathrm{m} \mu / \mathrm{l})$ \\
\hline Control $(\mathrm{n}=25)$ & $244 \pm 34.2$ & $5.4 \pm 0.4$ & $1.3 \pm 0.03$ \\
\hline All stages (75) & $707.8 \pm 36$ & $2.1 \pm 0.2$ & $1.2 \pm 0.02$ \\
\hline $\mathbf{P}^{?}$ & $<0.0001$ & $<0.0001$ & 0.1 \\
\hline Stage1 (n=10) & $249.8 \pm 29.6^{\mathrm{ns}}$ & $5 \pm 0.7^{\mathrm{ns}}$ & $1.32 \pm 0.02^{\mathrm{ns}}$ \\
\hline Stage2 $(\mathrm{n}=14)$ & $465.2 \pm 61.7^{* *}$ & $4.4 \pm 0.7^{\mathrm{ns}}$ & $1.31 \pm 0.03^{\mathrm{ns}}$ \\
\hline Stage3 $(\mathrm{n}=26)$ & $803.8 \pm 37.2^{* * *}$ & $1.1 \pm 0.4^{* * *}$ & $1.27 \pm 0.03^{\mathrm{ns}}$ \\
\hline Stage4 $(\mathrm{n}=13)$ & $852.4 \pm 67^{* * *}$ & $0.7 \pm 0.3^{* * *}$ & $1.14 \pm 0.05^{*}$ \\
\hline Stage 5 $(\mathrm{n}=12)$ & $1007.3 \pm 38^{* * *}$ & $1.3 \pm 0.04^{* * *}$ & $1.05 \pm 0.1^{* *}$ \\
\hline $\mathbf{P}^{\#}$ & $<0.0001$ & $<0.0001$ & 0.001 \\
\hline Early (stage1-2) & $607 \pm 41.6$ & $2.7 \pm 0.2$ & $1.3 \pm 0.01$ \\
\hline Late (stage 3-5) & $920.6 \pm 43$ & $1.02 \pm 0.1$ & $1.1 \pm 0.1$ \\
\hline $\mathbf{P}^{\$}$ & $<0.0001$ & $<0.0001$ & 0.005 \\
\hline
\end{tabular}

Data were expressed as mean \pm SE. $(*)$ significant, $\mathrm{p}<0.05,(* *)$ very significant, $\mathrm{P}<0.01$ and $(* * *)$ extremely significant, $\mathrm{p}<0.001$ and $(\mathrm{ns})$ not significant versus those of the control group. P?: p value of comparison between all stages vs. control

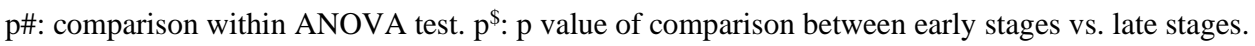

2. Correlation among NGAL, NO, and TAC and other markers.

In this study, there was a high negative correlation between NGAL and GFR ( $\mathrm{P}<0.0001, \mathrm{r}=-0.782)$. NGAL was negatively correlated with nitric oxide $(\mathrm{P}<$ $0.0001, \mathrm{r}=-0.547)$ and total-anti oxidant capacity $(\mathrm{p}=$ $0.006, r=-0.272$ ). On the other hand, a high positive correlation between NGAL and urea $(\mathrm{p}<0.0001, \mathrm{r}=$ 0.722). NGAL was positively correlated with creatinine $(\mathrm{p}<0.0001, \mathrm{r}=0.520)$ and uric acid $(\mathrm{p}<$ $0.0001, \mathrm{r}=0.529)$. Nitric oxide was highly positively correlated with GFR $(\mathrm{P}<0.0001, \mathrm{r}=0.669)$, TAC $(\mathrm{P}=$ $0.05, \mathrm{r}=0.196)$ but was negatively correlated with urea $(\mathrm{p}<0.0001, \mathrm{r}=-0.621)$, uric acid $(\mathrm{p}<0.0001, \mathrm{r}$
$=-0.357)$ and creatinine $(\mathrm{p}<0.0001, \mathrm{r}=0.344)$. A positive correlation between serum TAC and GFR $(\mathrm{p}=$ $0.001, r=0.331$ ) was found. On the contrary, GFR correlated negatively with creatinine $(\mathrm{p}=0.001, \mathrm{r}=$ $0.32)$ and urea $(\mathrm{p}=0.01, \mathrm{r}=-0.26)$.

\section{The diagnostic power of NGAL, NO, and TAC}

NGAL gave an AUC of 0.91 and those of $\mathrm{NO}$ and TAC were 0.83 and 0.58 , respectively, for discriminating patients with CKD from control subjects. NGAL was the most efficient index among other markers for discriminating patients with CKD from control subjects and discriminating patients in late stages from those in early stages (Table 3, figure1). 
Table 3. Diagnostic values of single markers to discriminate Chronic kidney disease (CKD) vs. control group and early stages versus late stages.

\begin{tabular}{|l|c|c|c|c|c|c|c|c|c|}
\hline Group & \multicolumn{7}{|c|}{ Control vs. patients } \\
\hline Parameters & Cut off & AUC & Sn & Sp & PPV & NPV & ACC & P \\
\hline NGAL & $\geq 296.85$ & 0.91 & $86.7 \%$ & $84 \%$ & $94.2 \%$ & $67.6 \%$ & $86 \%$ & $<0.0001$ \\
\hline TAC & $\geq 1.31$ & 0.58 & $52 \%$ & $60 \%$ & 79.6 & $29.4 \%$ & $54 \%$ & 0.2 \\
\hline NO & $\geq 3.88$ & 0.83 & $77.3 \%$ & $76 \%$ & $90.6 \%$ & $52.8 \%$ & $77 \%$ & $<0.0001$ \\
\hline Group & & & & & & & & & \\
\hline Parameters & Cut off & AUC & Sn & Sp & PPV & NPV & ACC & P \\
\hline NGAL & $\geq 785.5$ & 0.8 & $75 \%$ & $73 \%$ & $56.3 \%$ & $86 \%$ & $73.3 \%$ & $<0.0001$ \\
\hline TAC & $\geq 1.28$ & 0.75 & $66.6 \%$ & $66.7 \%$ & $48.5 \%$ & $81 \%$ & 66.7 & 0.001 \\
\hline NO & $\geq 0.74$ & 0.71 & $62.5 \%$ & $62.7 \%$ & $44.1 \%$ & $78 \%$ & $62.7 \%$ & 0.003 \\
\hline
\end{tabular}

AUC: Area under the ROC curve. Sp: Specificity. Sn: Sensitivity. PPV: Positive predictive value. NPV: Negative predictive value. $\mathrm{P}$ value: $\mathrm{P}>0.05$ non significant $. \mathrm{P}<0.05$ : Significant. $\mathrm{P}<0.001$ : very significant. $\mathrm{P}<0.0001$ : Extremely significant. 

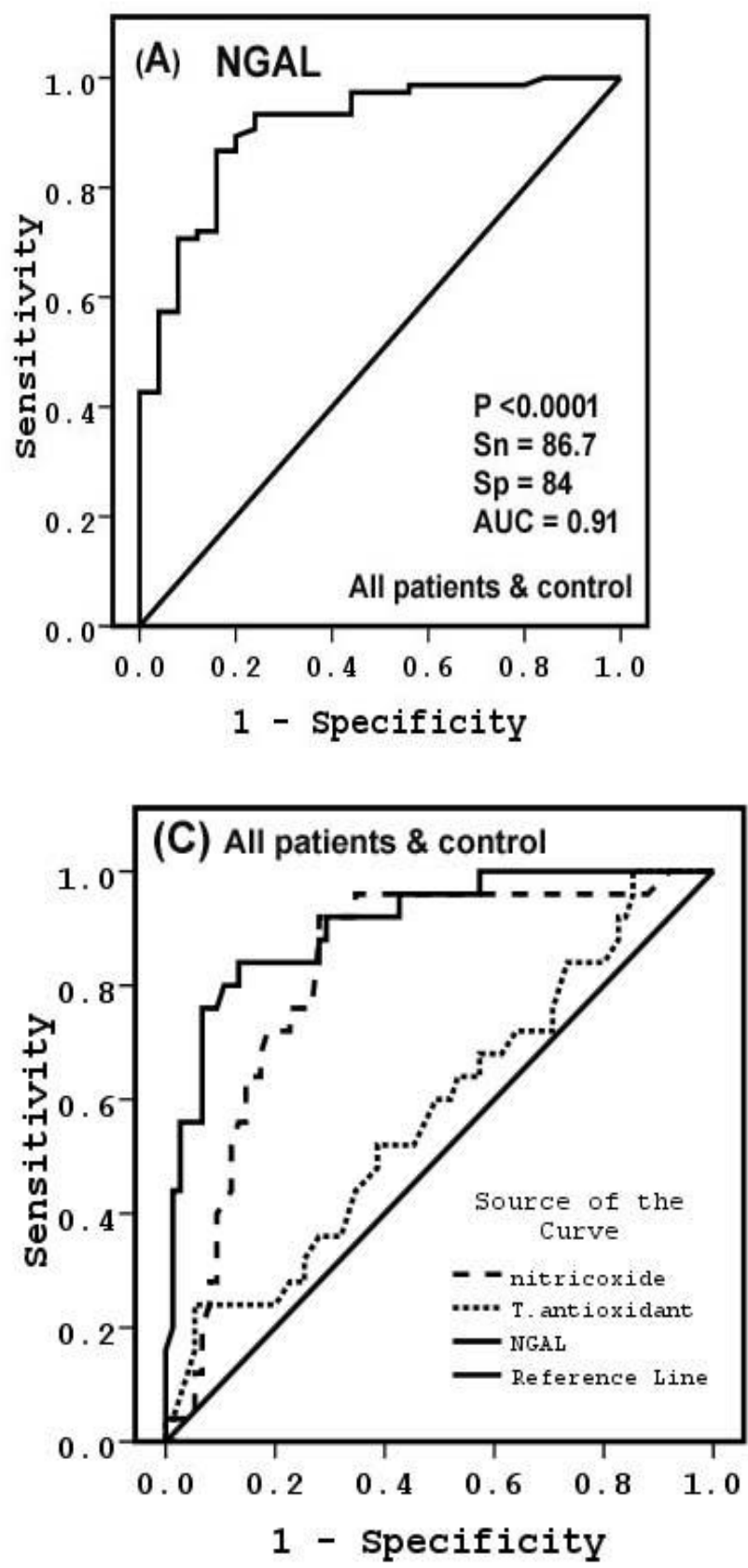
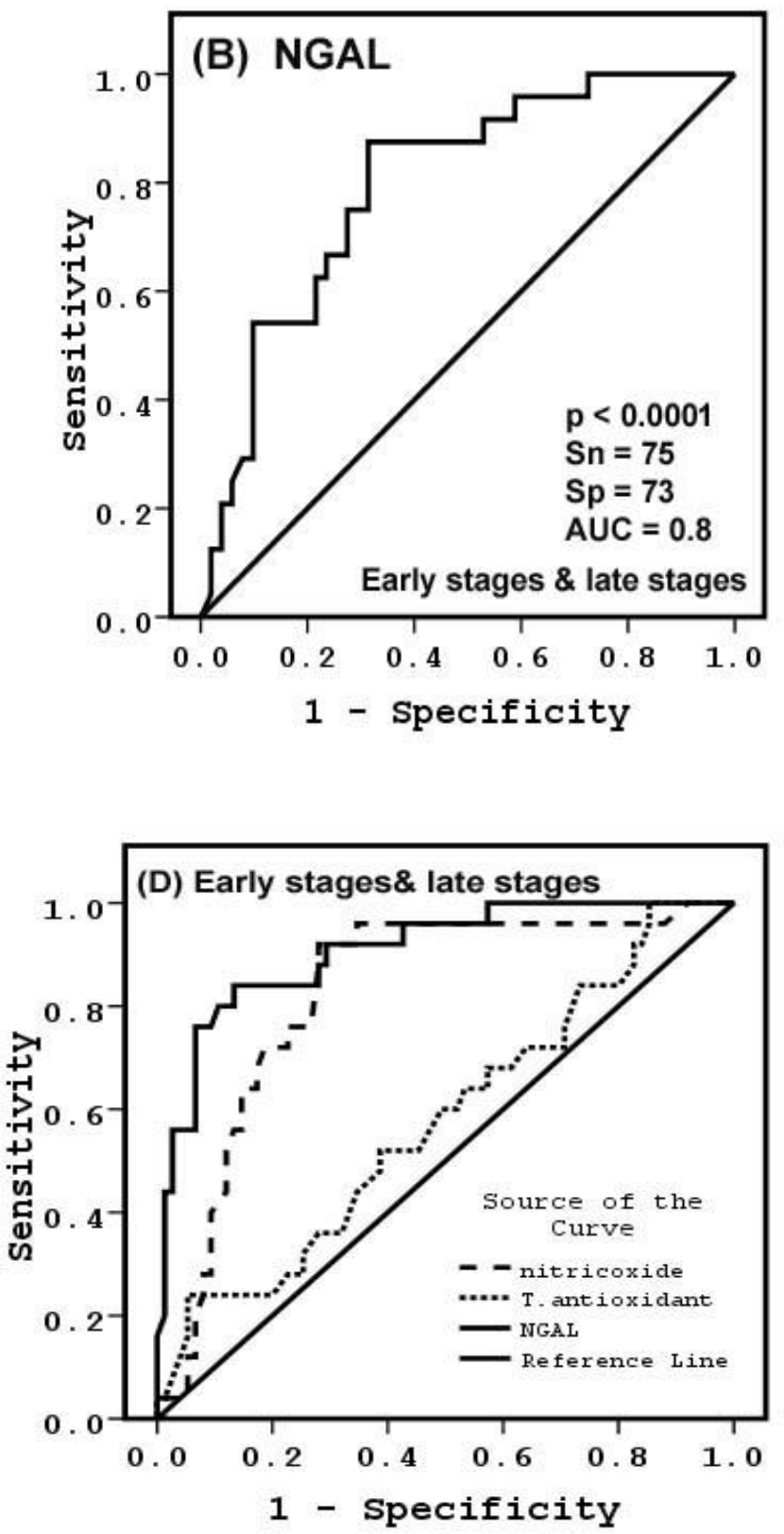

Figure 1: AUC generated by NGAL(A) for discriminating all patients with CKD from those in control and (B) for discriminating patients with CKD in late stages from those in early stages. (C): AUC for NGAL compared with those of NO and TAC for discriminating all patients with CKD from those in control and $(D)$ for discriminating patients with CKD in late stages from those in early stages. 


\section{The diagnostic power of combined markers}

The combination of (NGAL and NO), (NGAL and TAC), (NO and TAC) (NGAL+TAC+NO) yielded AUCs of $0.93,0.90,0.80$ and 0.92 for discriminating patients with CKD from control subjects; respectively, and $0.82,0.84,0.80$ and 0.84 for discriminating patients in late stages from those in early stages; respectively. The best linear combination of blood markers was selected by the multivariate discriminate analysis for the development $\mathrm{NN}$ score $=1.550$ (numeric constant) $+0.001(\mathrm{NGAL})(\mathrm{ng} / \mathrm{ml})+(-0.042)(\mathrm{NO})(\mu \mathrm{mol} / \mathrm{l})$. This score was based on NGAL $(\mathrm{AUC}=0.91)$ as the backbone followed by NO $(\mathrm{AUC}=0.83)$. Their combination (NN score) gave an AUC of 0.93 and a discriminate cut off $>1.7$. Its sensitivity, specificity, the positive predictive and negative predictive values were $84 \%, 84 \%, 94 \%$, and $63.6 \%$; respectively $(\mathrm{p}<0.0001)$, when differentiating patients with CKD from control subjects (Figure 2A and Table 4). There is another score, namely (NT) score, which calculated according to $\mathrm{NT}$ score $=1.780$ (numeric constant) +0.001 (NGAL) $(\mathrm{ng} / \mathrm{ml})+(-0.716)(\mathrm{TAC})(\mathrm{m} \mu / \mathrm{l})$. NGAL was the backbone of this score due to its highest AUC (0.80) followed by TAC (AUC $=0.75)$ for discriminating patients in late stages from those in early stages with an AUC of 0.84 at a discriminate cut off $>1.17$ ( $<<0.0001$, Figure $2 \mathrm{~B}$ and Table 4), with $75 \%$ sensitivity and $74.5 \%$ specificity. The positive predictive and negative predictive values were $58.1 \%$ and $86.4 \%$; respectively.

Table 4. Diagnostic performance of combined markers for discriminating control group versus all patients with CKD and early stages versus late stages.

\begin{tabular}{|l|c|c|c|c|c|c|c|c|}
\hline Group & \multicolumn{7}{|c|}{ Control vs. patients } \\
\hline Parameters & AUC & Cut off & Sn & Sp & PPV & NPV & ACC & P \\
\hline NGAL+NO & 0.93 & $\geq 1.72$ & $84 \%$ & $84 \%$ & $94 \%$ & $63.6 \%$ & $84 \%$ & $<0.0001$ \\
\hline NGAL+TAC & 0.90 & $\geq 1.675$ & $82.7 \%$ & $84 \%$ & $93.9 \%$ & $61.8 \%$ & $83 \%$ & $<0.0001$ \\
\hline TAC+NO & 0.79 & $\geq 1.83$ & $73.3 \%$ & $73 \%$ & $86.7 \%$ & $47.7 \%$ & $73 \%$ & $<0.0001$ \\
\hline NGAL+TAC+NO & 0.92 & $\geq 1.656$ & $84 \%$ & $84 \%$ & $94 \%$ & $63.6 \%$ & $84 \%$ & $<0.0001$ \\
\hline Group & & & & & & & & \\
\hline Parameters & AUC & Cut off & Sn & Sp & PPV & NPV & ACC & P \\
\hline NGAL+NO & 0.82 & $\geq 1.51$ & $70.8 \%$ & $72.5 \%$ & $54.8 \%$ & $84.1 \%$ & $72 \%$ & $<0.0001$ \\
\hline NGAL+TAC & 0.84 & $\geq 1.70$ & $75 \%$ & $74.5 \%$ & $58.1 \%$ & $86.4 \%$ & $74.7 \%$ & $<0.0001$ \\
\hline TAC+NO & 0.80 & $\geq 1.33$ & $75 \%$ & $73.5 \%$ & $56.3 \%$ & $86 \%$ & $73.3 \%$ & $<0.0001$ \\
\hline NGAL+TAC+NO & 0.84 & $\geq 1.75$ & $75 \%$ & $74.5 \%$ & $58.1 \%$ & $86 \%$ & $74.7 \%$ & $<0.0001$ \\
\hline
\end{tabular}

AUC: Area under the ROC curve. Sp: Specificity. Sn: Sensitivity. PPV: Positive predictive value. NPV: Negative predictive value. $\mathrm{P}$ value: $\mathrm{P}>0.05$ non significant. $\mathrm{P}<0.05$ : Significant. $\mathrm{P}<0.001$ : very significant. $\mathrm{P}<0.0001$ : Extremely significant. 

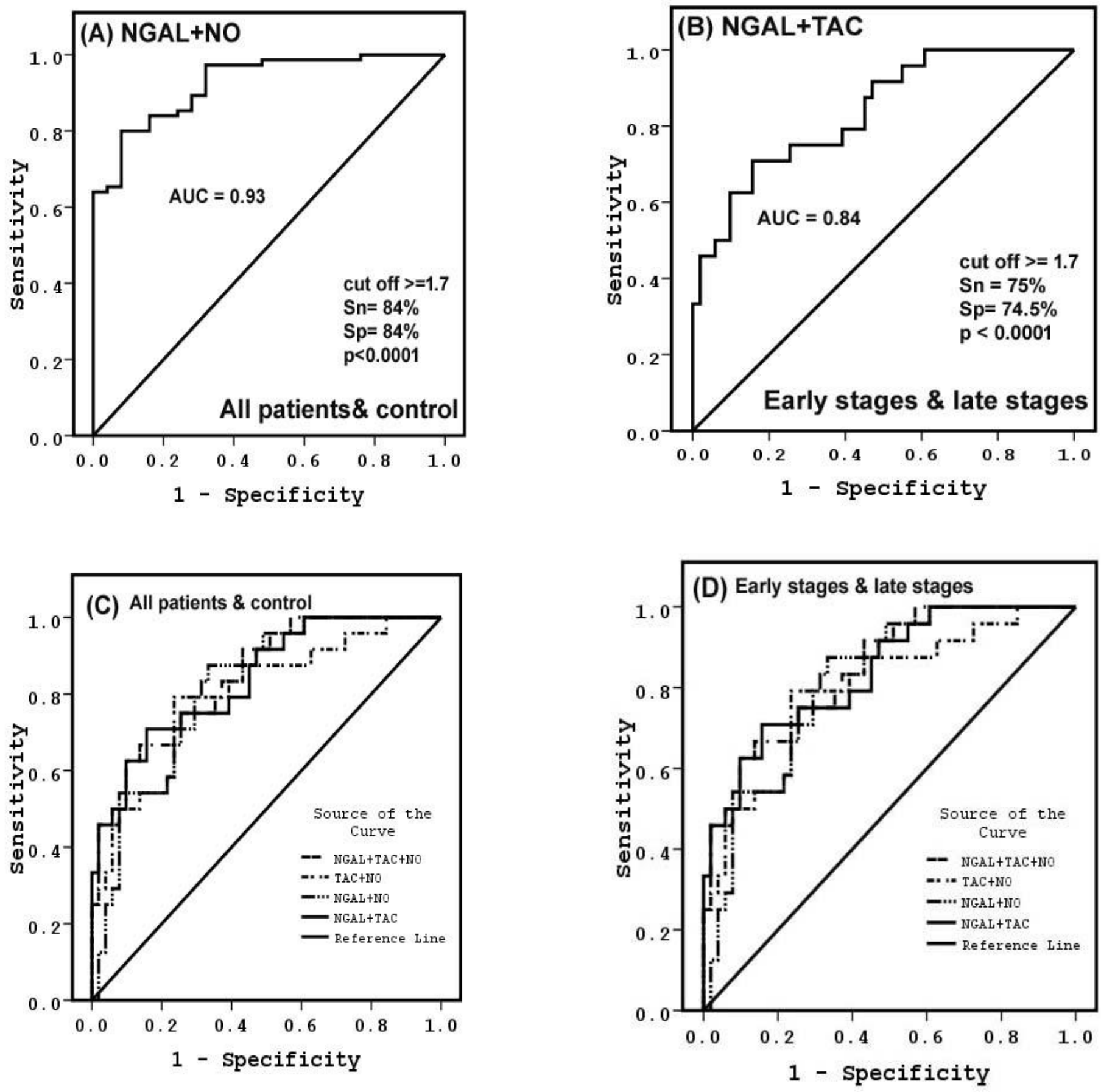

Figure2: Receiver-operating characteristic curves (ROC) of NGAL+NO (NN score) for discriminating all patients with CKD from control subjects (A) and ROC of NGAL +TAC (NT) for differentiating patients in late stages from those in early stages (B). Area under the ROC curve for the NN score and comparison with other scores for discriminating patients with CKD from control subjects (C) and area under the ROC curve for NT score for differentiating patients in late stages from those in early stages (D). 


\section{Discussion}

Patients with CKD commonly develop different complications. Now, CKD has become major public health trouble. Moreover, a dramatically increased overall prevalence of CKD was reported, especially for earlier stages (Coresh et al., 2007). Traditional biomarkers such as creatinine or/and proteinuria, are not sufficient to fully explain the pathophysiology of the disease and to predict the progression of CKD.

In the present study, 24 cases $(32 \%)$ were in late stages and about $50 \%$ (12 cases) were in stage 5 of CKD and 51 cases $(68 \%)$ were in the early stages of CKD. The level of NGAL increases progressively with increase CKD severity, especially in stage 5 of the disease. The probable explanation for the elevated level of NGAL is its expulsion from the damaged tubular tissues of the kidney into a serum. Farther NGAL has a role in up-regulation and promotion of nephrotic apoptosis (Chakraborty et al., 2012). These results agreed with that of Bolignano et al (2009), they showed that the NGAL levels of late CKD were higher than those of the early CKD group and the levels of both groups were higher than those of the control subjects. In the present study, NGAL gave an AUC of 0.91 for differentiating patients with CKD from those with control with $86.7 \%$ sensitivity, $84 \%$ specificity, 94\% PPV, and 67.6\% NPV. Somewhat similar results were reported by Avic et al (2016). In their results, the plasma NGAL levels of patients with CKD were higher than that of the control $(\mathrm{p}<0.0001)$. Moreover, GAL gave an AUC of 0.8 for differentiating patients with late CKD from those with early stages of CKD. The sensitivity, specificity, PPV, and NPV were only $75 \%, 73 \%, 56.3 \%$, and $86 \%$; respectively. The results of Xiang et al (2014), gave an AUC >0.8, and >0.7 as well as specificity $>87 \%$ and $>90 \%$ for serum and urine NGAL, respectively. In this study, there is a high negative correlation between NGAL and GFR (P < $0.0001, r=-0.782)$. Similar findings were found by Liu et al (2013) and Bastruk et al (2017).

Renal sources of ROS are cells of renal tissue like vascular (endothelial and smooth muscle cells), glomerular (endothelial and mesangial cells), tubular (proximal, distal, and convoluted tubular cells) (Gonzalez, 2003) along with infiltrating blood cells and infection controlling cells like neutrophils, macrophages, and phagocytes. Thus, hyper-production of ROS was expected to be associated with shortened cell survival, deformed cells leading to lipid peroxidation. Thus, one can expect that CKD patients are characterized by oxidative stress, which causes inflammation and a reduction in the nitric oxide level (Cachofeiro et al., 2018). The reduction in the level of total antioxidant capacity, in the present study, with the increase in the severity of CKD leads one to propose the involvement of free radicals in the pathogenesis of such disease. These results not only confirm those of Shivprasad et al (2013) and data reviewed by Modaresi et al (2015) but also those of Reshma et al (2017) who reported that antioxidant levels, as measured by total antioxidant capacity, was found to be significantly lower in CKD patients and they argue this for the possible imbalance in patient's antioxidant status with their oxidants which finally mediate oxidative stress and CKD. Nitric oxide is synthesized by the kidney and it plays an important role in blood pressure regulation. Decrease level of NO in early CKD stages can be explained due to a decrease in the endogenous L-arginine production, which is mainly synthesized in the kidney and due to a reduction in its transportation into cells to the vicinity of the nitric oxide synthase. Thus, one cannot neglect the involvement of the reduction of the serum NO level in the progression of CKD, which was observed in patients of our study. Surprisingly, NO was re-increased, especially in stage 5 because this stage includes 8 patients under dialysis. In this regard, Mounuddin, and Laxmikanth (2017) argue the increase in NO production to haemoincompatibility of the dialyzing membrane. Also, the increase in the blood turbulence and shearing of stress, due to the mechanical components of the dialyzer, can activate both the platelets as well as leukocytes. The later activation augments the excessive release of NO from them. Moreover, the use of heparin may be 
another factor in this enhanced production. In the present study, an AUC of only 0.83 for NO was found to discriminate between patients with CKD and healthy individuals (table3). Therefore, its individual values were added to those of NGAL. The result of this combination yielded an AUC of 0.93, the sensitivity of $84 \%$, a specificity of $84 \%$, PPV of 63.6 , NPV of $94 \%$, and accuracy of $84 \%$. Based on the first letter of each of the two markers, this score was called $\mathrm{NN}$ score= 1.550 (numeric constant) +0.001 (NGAL) $(\mathrm{ng} / \mathrm{ml})+(-$ 0.042) (NO) $(\mu \mathrm{mol} / \mathrm{l})$. The NGAL with its highest AUC was used to be the backbone of the selected NN score. The AUC of TAC was only 0.58 , so it was excluded from the possible combinations for that discrimination. In the present study, the AUC of NO (0.71) was the lowest between other markers, so it was excluded from the possible combinations for discriminating late stages from those in the early stages of the disease. The AUC of NGAL and TAC combination was 0.84 with a sensitivity of $75 \%$, a specificity of $74.5 \%$, PPV of 58.1 , NPV of 86.4 , and accuracy of $76.7 \%$ for discriminating patients with late stages from those in early stages. Based on the first letter of each of these markers, this score is termed NT and calculated according to NT score $=1.780$ (numeric constant $)+0.001(\mathrm{NGAL})(\mathrm{ng} / \mathrm{ml})+(-0.716)(\mathrm{TAC})$ $(\mathrm{m} \mu / \mathrm{l})$, which can now be used to reflect the severity of chronic kidney diseases and their differentiation.

In conclusion, the use of NGAL, NO, and TAC based scores can now help in improving the diagnostic efficacy of CKD. This is because NGAL reflects tubular damage, $\mathrm{NO}$ is an angiogenic marker as well as an oxidative stress marker. Beside, TAC adds more to the involvement of oxidative stress in the establishment of chronic kidney disease.

\section{Abbreviations}

NGAL, Neutrophil gelatinase-associated lipocalin; NO, Nitric oxide; TAC, total antioxidant capacity; MDA, multivariate discriminant analysis; NNT, NGAL- Nitric oxide- Total antioxidant capacity; ROC, receiver operating characteristic; NOS, NO synthase; eNOS, endothelial NOS; iNOS, inducible
NOS; GFR, glomerular filtration rate; Sn, sensitivity; $\mathrm{Sp}$, specificity; PVP, the predictive value of positive; PVN, predictive value of negative; BUN, blood urea nitrogen

\section{References}

Avci Çiçek E, Rota S, Dursun B and Kavalci E. 2016. Evaluation of serum NGAL and hepcidin levels in chronic kidney disease patients. Ren Fail; 38 (1): 35-39.

\section{Basturk T, Sari O, Koc Y, Eren N, Isleem M, Kara} E, Sevinc M, Sakaci T, Ahbap E, Hasbal NB, Bayrakdar Caglayan $F$ and Unsal A. 2017. Prognostic significance of NGAL in early stage chronic kidney disease. Minerva Urol Nefrol; 69 (3): 307-312.

Baylis C. 2008. Nitric oxide deficiency in chronic kidney disease. Am J Physiol Renal Physiol; 294 (1):F1-F9.

Bolignano D, Donato V, Coppolino G, Campo S, Buemi A, Lacquaniti A and Buemi M. 2008. Neutrophil gelatinase-associated lipocalin (NGAL) as a marker of kidney damage, Am J Kidney Dis; 52(3): 595-5605.

\section{Bolignano D, Lacquaniti A, Coppolino G, Donato} V, Campo S, Fazio MR, Nicocia G and Buemi M. 2009. Neutrophil gelatinase-associated lipocalin (NGAL) and progression of chronic kidney disease. Clin J Am Soc nephrol; 4 (2): 37-44.

Cachofeiro V, Goicochea M, de Vinuesa SG, Oubiña P, Lahera $V$ and Luño J. 2008. Oxidative stress and inflammation, a link between chronic kidney disease and cardiovascular disease, Kidney Int Suppl;111: S4-S9.

Chakraborty S, Kaur S, Guha S and Batra SK. 2012. The multifaceted roles of neutrophil gelatinase associated lipocalin (NGAL) in inflammation and cancer. Biochim Biophys Acta; 1826 (1):129-169.

Coresh J, Selvin E, Stevens LA, Manzi J, Kusek JW, Eggers P, Van Lente F and Levey AS. 
2007. Prevalence of chronic kidney disease in the United States, JAMA; 298 (17): 2038-2047.

Gonzalez B. 2003. Progression of chronic renal failure and oxidative stress. J Biomed; 1(1): 5-11.

Kjeldsen L, Johnsen AH, Sengeløv $H$ and Borregaard N. 1993. Isolation and primary structure of NGAL, a novel protein associated with human neutrophil gelatinase. J Biol Chem; 268 (14):1042510432.

Levey AS, Stevens LA, Schmid CH, Zhang YL, Castro AF, Feldman HI, Kusek JW, Eggers P, Van Lente F, Greene T and Coresh J. 2009. A new equation to estimate glomerular filtration rate. Ann Intern Med; 50 (9): 604-612.

Liu KD, Yang W, Anderson AH, Feldman HI, Demirjian S, Hamano T, He J, Lash J, Lustigova E, Rosas SE, Simonson MS, Tao K and Hsu CY. 2013. Urine neutrophil gelatinase-associated lipocalin levels do not improve risk prediction of progressive chronic kidney disease. Kidney Int; 83(5): 909-914.

Modaresi A, Nafar $M$ and Sahraei Z. 2015. Oxidative Stress in chronic kidney disease. Iran $\mathbf{J}$ Kidney Dis; 9 (3):165-179.

Modaresi A, Nafar M and Sahraei Z. 2015. Oxidative stress in chronic kidney disease. Iran $\mathbf{J}$ Kidney Dis; 9 (3):165-179.

Mori K and Nakao K. 2007. Neutrophil gelatinaseassociated lipocalin as the real-time indicator of active kidney damage. Kidney Int; 71(10): 67-70.
Mounuddin M and Laxmikanth B. 2017. Serum nitric oxide levels in chronic renal failure patients on maintenance hemodialysis. Int J Bio Research; 8(10): 576-579.

Reshma S, Kavitha A Kumar, Soundarya, Ashok Kumar J, Sushith, Prathima M and Madan Gopal Rajan. 2017. Oxidative stress markers in chronic kidney disease patients with hearing loss. Int J Bio Research; 8(5): 259-262.

Shivprasad S, Smita Sonoli and Anuradha B. 2013. Oxidative stress and total antioxidant capacity in renal failure and renal calculi patients-A cross sectional study. Nat J Lab Med; 2 (1): 1-4.

Thambyrajah J, Landray MJ, McGlynn FJ, Jones HJ, Wheeler DC and Townend JN.2000. Abnormalities of endothelial function in patients with predialysis renal failure. Heart; 83( 2): 205-209.

Wever R, Boer P, Hijmering M, Stroes E, Verhaar M, Kastelein J, Versluis K, Lagerwerf F, van Rijn H, Koomans H and Rabelink T. 1999. Nitric oxide production is reduced in patients with chronic renal failure. Arterioscler Thromb Vasc Biol;19 (5):11681172.

Xiang D, Zhang H, Bai J, Ma J, Li M, Gao J and Wang C. 2014.

Clinical application of neutrophil gelatinaseassociated lipocalin in the revised chronic kidney disease classification. Int J Clin Exp Pathol; 7(10): 7172-7181. 